\title{
MATHEMATICAL MODELING AND NUMERICAL ANALYSIS OF FORCE MONITORING OF FOUNDATION PIT SUPPORT STRUCTURE BASED ON VIBRATION RESPONSE SENSOR SYSTEM
}

\author{
Yongqiu Xu \\ Chongqing City Vocational College, Chongqing 402160, China \\ Email: xuyongqiu4021@163.com
}

\begin{abstract}
In order to study the stability and safety of the foundation pit supporting structure system, the wireless tilting vibration sensor is used to discuss the underground monitoring of the underground engineering, the horizontal displacement monitoring of the foundation pit support and the deep foundation pit supporting structure. The actual measured data is compared with the mathematical simulation values. The results show that the foundation pit engineering and shield tunnel engineering can use the wireless vibration tilt sensor for underground engineering monitoring. The width of the foundation pit and the way of excavation are the main factors affecting the internal force and stability of the steel support. With the layered excavation of the foundation pit, the deformation of the retaining structure also has obvious segmental features. The sub-section excavation according to the space-time effect can effectively reduce the lateral displacement of the retaining structure. Therefore, for the structural characteristics of underground engineering, the wireless vibration tilt sensor and the method of finite element numerical simulation are combined. The joint support system of steel support and retaining pile has a coordination relationship between deformation and force. The steel support can effectively control the deformation of the envelope structure. When the steel support is erected and $60 \%$ of the design axial force value is added, the deformation of the retaining structure can be better reduced. Furthermore, the safety and stability of the structure is increased.
\end{abstract}

Keywords: Wireless Vibration Tilt Sensor; Foundation Pit Supporting Structure; Finite Element Numerical Simulation.

\section{Introduction}

In recent years, with the rapid development of China's economy and the continuous progress of urbanization, the investment in the development of urban underground space and the construction of transportation infrastructure has been continuously deepened. The development and construction of underground engineering has become an important part of urban construction in China [1]. At present, the safety monitoring of underground structures during construction and operation mainly adopts traditional manual monitoring and wired automatic monitoring. Generally, manual monitoring is costly and has low precision [2]. More importantly, the monitoring data is not continuous, which leads to a great decline in the ability of responding, handling and warning to emergencies.

In this context, the application of wireless vibration response sensing technology in foundation pit and tunnel construction and health monitoring, operation and maintenance is studied for the technical defects of current safety monitoring methods in underground engineering $[3,4]$.

Through the application of specific demonstration projects, the application effect of wireless vibration sensing technology in underground engineering monitoring is verified. The mechanical behavior of the support system is studied and analyzed by numerical simulation.

The urban tunnel project is located in a prosperous road section with a large number of dense underground pipelines [5]. The excavation width of the foundation pit is $19.9 \mathrm{~m} \sim 27.9 \mathrm{~m}$, and the deepest depth is $18.6 \mathrm{~m}$. Due to the width and depth of the excavation of the foundation pit, the influence on the adjacent building is large. According to the previous engineering experience, the foundation pit support structure adopts a cast-in-place pile, and an internal support structure is erected inside.

This kind of combined support system is widely used in the construction of urban underground tunnels and roadways whose width is far less than the length of the foundation pit [6]. 
This is a supporting structure system that is economical and can better control structural deformation. The inside of the foundation pit adopts a steel support structure. Compared with the concrete support, the steel support structure has the advantages of erection, easy removal and recyclability.

The biggest advantage is that it can pre-add axial force according to the deformation of the retaining structure. It is an economical and effective internal support structure, which is widely used in the support system with retaining pile and internal support. In addition, the steel support also has the characteristics of simple connection and clear force. Through the monitoring of the axial force of the steel support and the change law of the deformation of the retaining structure, it can be known that the deformation of the foundation pit retaining structure and the axial force of the steel support change with the progress of the foundation pit excavation [7].

Further, the safety of the pit structure is effectively judged. The monitoring of the support axial force and the deformation of the retaining structure is an important part of the comprehensive monitoring of the foundation pit engineering. By analyzing the interaction relationship between the two with time, it is of great significance to accurately and comprehensively judge the safety and stability of the foundation pit [8].

The three-dimensional simulation analysis of the deep foundation pit support system was carried out by using finite element analysis software. Throughout the construction phase, the relationship between the variation of the support axial force and the deformation of the retaining structure and the supporting system under various working conditions is calculated. The simulated and measured data results are compared.

This provides a reference for the construction optimization of similar projects, the prediction of monitoring results and similar engineering numerical simulations.

\section{Literature Review}

Terzaghi and Peck put forward the estimation method of excavation stability and the total stress method to calculate the support load. Terzaghi's soil mechanics theory is used to analyze the force of soil on the supporting structure in the foundation pit design for the design and stability analysis of the supporting structure [9]. The proposed method provides cross-age significance for the research and analysis of foundation pit engineering, and the principle has been used until now.

By reducing the support spacing, the stiffness of the entire support system can be greatly increased. Moreover, the lateral and vertical steel support stiffness is equally important to the overall safety of the support system [10].

According to the field experiment, the effect of bolt support on pile row and the effect of bolt on the earth pressure distribution behind the wall are analyzed. The method of anchor design and the basic assumptions for establishing the model are proposed.

For the first time, the "intermediate active state" of the soil was proposed [11]. An active earth pressure expression for the rotation of the wall toe is established to estimate the lateral earth pressure behind the rigid retaining wall.

According to the generalized displacement control criterion, the variation of the displacement of the top of the rod under the action of the unit force is proposed.

In 1997, a bridge environment and performance evaluation system based on wireless sensing technology was developed. The wireless sensor nodes in the evaluation system include induction unit, battery unit, small transponder and microprocessing unit. The regional controller of the wireless evaluation system is placed on the bridge bank, and other wireless sensor nodes are placed on the bridge [12]. The sensor node has the data acquisition function.

In 1999, some computing power was transferred to the sensor board, which was called the fourthgeneration sensor. This generation of sensors has bidirectional command and digital communication, self-query, online storage, local data processing, preprogramming algorithm, compensation algorithm, user-defined algorithm, complete digital transmission, extensible sensor object model and other functions [13].

In 1999, a structural intelligent monitoring acquisition system based on wireless sensing technology was developed. The system uses a multiplexer to perform data collection for each sensing unit [14]. The sensing unit data is transmitted through a single wireless channel. The Internet network expansion of the acquisition system was carried out.

\section{Methodology}

\subsection{Principles of wireless monitoring technology for underground engineering}

The wireless vibration tilt sensor is a micro sensor composed of a gyroscope or a single-multiaxis acceleration sensor. When monitoring the tilt of the system, a common method of the wireless vibration tilt sensor is to integrate the output of the gyroscope.

Although this method is straightforward, the error associated with zero-point bias stability 
accumulates rapidly as the integration period increases, causing significant rotation of the device.

In some applications, if the net acceleration or force acting on the system over the entire time range is gravity, an accelerometer can be used to measure the static angle of the tilt [15]. For tilt monitoring with accelerometers, gravity is assumed to be the only acceleration stimulus. In actual use, the gravity vector and its projection on the accelerometer axis are used to determine the tilt angle.

The tilt sensor used herein is a static $\mathrm{H}$-axis tilt sensor consisting of an accelerometer. The tilt angle is determined by gravity vector and its projection on the axis of the accelerometer. According to the basic $\mathrm{H}$-angle equation, when the accelerometer axes of the sensor are tilted, the gravity vector projection of the axis produces an output acceleration. Its size is equal to the sine of the angle between the accelerometer axis and the horizontal plane.

\subsection{Principle of horizontal displacement monitoring of foundation pit support based on wireless vibration inclination sensor}

The "Technical Specifications for Inspection of Foundation Pit Engineering of 50497-2009" states: The horizontal displacement of the retaining wall should be monitored by embedding the inclined pipe in the wall or soil and observing the horizontal displacement at various depths with the inclinometer. At the same time, the horizontal displacement and height difference between the top and the bottom of the monitored object should be measured in the building tilt monitoring. The obliquity, obliquity direction and obliquity rate of the monitored object were recorded and calculated respectively.

Based on the principle of monitoring the displacement of foundation pit support based on wireless vibration inclination sensor, the principle of deep horizontal displacement monitoring of foundation pit is combined with the principle of building inclination monitoring. The horizontal displacement monitoring section of each foundation pit support structure is divided into several monitoring sections in the vertical direction. Each monitoring segment is provided with a wireless vibration inclination sensor. The inclination data measured by the wireless vibration tilt sensor can be converted into a horizontal displacement relative to the reference point using a geometric relationship.

Then, combined with the reference point horizontal displacement monitoring value, the horizontal absolute displacement of the foundation pit support is determined. The main purpose of the convergence meter monitoring is to monitor the change of the pipe diameter at the key position of the shield tunnel lining ring to grasp the dynamic deformation of the entire monitoring ring segment.
In the actual monitoring activities, the monitoring of the vertical diameter and other azimuth diameters is not suitable for monitoring with the convergence meter due to the convergence of the transverse diameter. In addition to the diameter detection, the change in the inclination of the segment can also reflect the dynamic changes of the entire monitoring ring segment. The combination of tilt sensor and convergence monitoring is used to reflect the dynamic changes of the entire monitoring loop.

The principle of shield tunnelling deformation monitoring based on wireless tilt sensor is based on the following assumptions: The deformation form is represented by the rotation and translation of the segment, and the deformation of the segment itself is neglected, that is, the segment is a rigid body. Since the angle of rotation of the segments is small, the azimuth angle of each longitudinal slit remains unchanged. At the arch waist $\left(0^{\circ}\right.$ and $\left.270^{\circ}\right)$, the tube convergence monitoring point is also in rotation.

\subsection{Numerical simulation of horizontal position of typical support structure of foundation pit engineering}

In order to explore the influence of the position of the foundation pit on the horizontal displacement of the supporting structure, four working conditions are set according to the supporting position and the number.

The supporting conditions of each working condition are shown in Table 1.

Table 1 Support conditions for each working condition

\begin{tabular}{|c|c|c|}
\hline $\begin{array}{c}\text { Project } \\
\text { number }\end{array}$ & $\begin{array}{c}\text { Support } \\
\text { quantity }\end{array}$ & Support position \\
\hline 1 & 1 & $0 \mathrm{~m}$ \\
\hline 2 & 2 & $0 \mathrm{~m},-6 \mathrm{~m}$ \\
\hline 3 & 3 & $0 \mathrm{~m},-3 \mathrm{~m},-6 \mathrm{~m}$ \\
\hline 4 & 4 & $0 \mathrm{~m},-3 \mathrm{~m},-6 \mathrm{~m},-9 \mathrm{~m}$ \\
\hline
\end{tabular}

The self-weight load applied by each working condition is solved by linear static force, and the normal displacement constraint is applied around the soil mesh and the bottom surface.

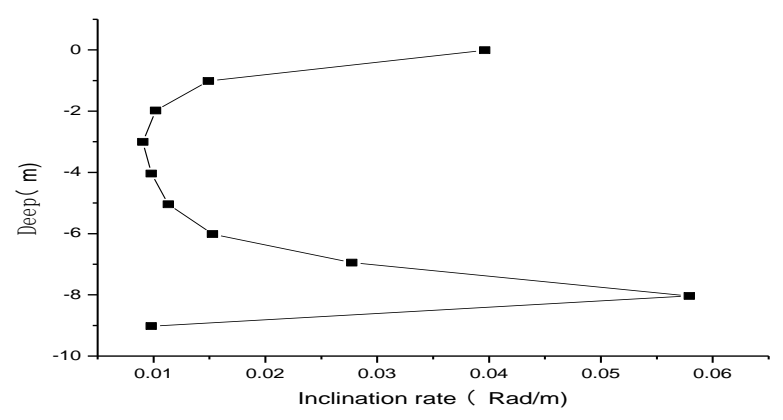

Figure 1: Diagram of inclination value of enclosure wall under condition 1 


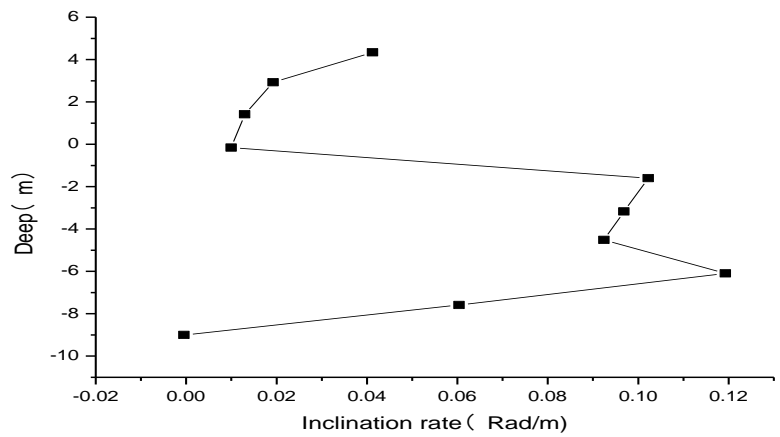

Figure 2 Diagram of inclination value of enclosure wall under condition 2

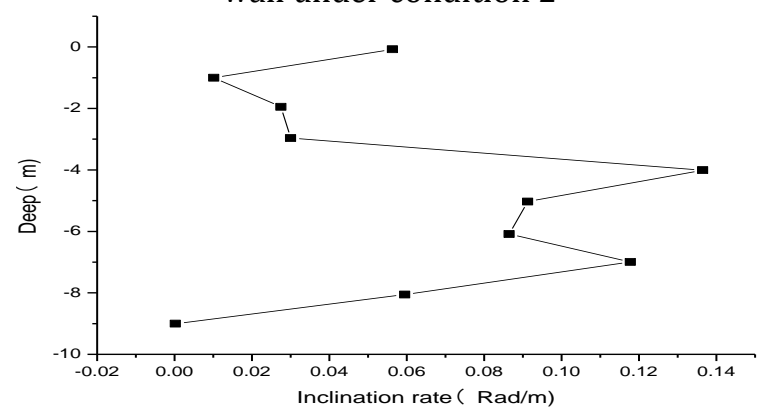

Figure 3 Diagram of inclination value of enclosure wall under condition 3

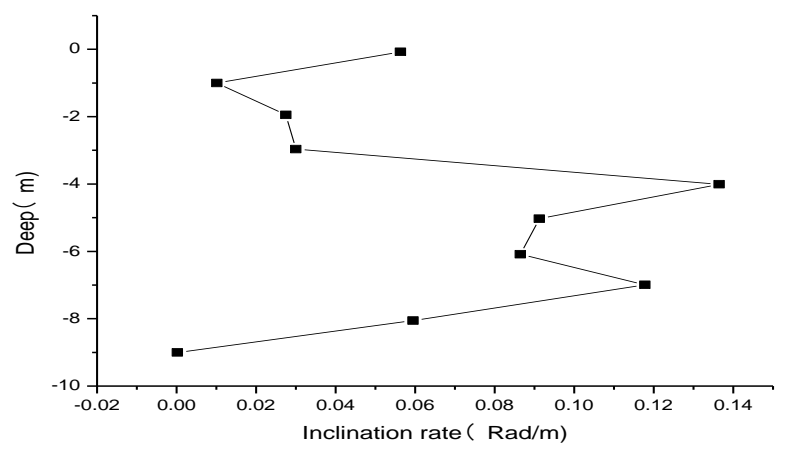

Figure 4: Diagram of inclination value of enclosure wall under condition 4

According to the curve of the value of the inclination angle of the retaining wall under four working conditions, the change rate of the inclination angle of the supporting position of the retaining wall is larger than that of the unsupported position.

According to the above numerical simulation results of the foundation pit support structure, the following optimization suggestions are proposed for the layout scheme of the wireless vibration inclination sensor in the horizontal displacement monitoring of the foundation pit support:

The monitoring points of the wireless vibration inclination sensor should be coincident with the monitoring points of the horizontal displacement on the top of the artificial support structure as far as possible.

The vertical arrangement of the wireless vibration tilt sensor in the monitoring point enclosure structure should be avoided from being directly placed at the joint between the support and the enclosure structure. The layout density of the wireless vibration tilt sensor should be increased in the vicinity of the connection between the support wall and the support structure.

The top and bottom of the retaining wall shall be equipped with wireless vibration inclination sensor.

Under the condition that the 2, 3, and 4 wireless vibration tilt sensor layout optimization suggestions are met, the wireless vibration tilt sensor should be arranged vertically in the vertical position of the monitoring point. For a longer distance, the unsupported interval can be appropriately widened to reduce the monitoring cost.

\subsection{Numerical analysis of deformation of support structure in foundation pit engineering}

The finite element model is simulated by construction conditions. The working condition step setting is set according to the foundation pit construction organization plan, as shown in Table 2 .

Table 2 Steps of construction condition

\begin{tabular}{|c|c|c|c|c|}
\hline No. & Working condition name & Excavation depth $(\mathrm{m})$ & Excavation area & Activation structure \\
\hline 1 & Original ground stress & 0 & - & \\
\hline 2 & Excavation 1 & 1 & Complete pit area & $\begin{array}{c}\text { Envelope structure, one- } \\
\text { layer support }\end{array}$ \\
\hline 3 & Excavation 2 & 4.5 & Complete pit area & Two layers support \\
\hline 4 & Excavation 3 & 7.25 & CDFGIJ & \\
\hline 5 & Excavation 4 & 9.25 & CDFGIJ & \\
\hline
\end{tabular}

The numerical simulation results of the finite element model of foundation pit excavation show that the horizontal displacement and deformation of the retaining wall towards the inner direction of the foundation pit gradually increase with the excavation degree.
The horizontal deformation of the retaining wall at the test point of the horizontal displacement monitoring test of the foundation pit support structure with the wireless vibration inclination sensor presents a parabolic shape as a whole. 


\subsection{Comparison of the results of wireless monitoring and manual testing}

The monitoring data of horizontal displacement of foundation pit support with wireless vibration inclination sensor show that the horizontal displacement of the monitoring section at the bottom and top of the foundation pit is small. The overall deformation trend of foundation pit support structure at the horizontal displacement monitoring section is parabolic. The test foundation pit support structure has a large horizontal displacement in the middle of the horizontal displacement monitoring section. The horizontal displacement at the monitoring section -3 meters reached $346 \mathrm{~mm}$ on April 7. The total horizontal displacement of the deep foundation pit support was $0.59 \mathrm{~mm}$ during the 3 -week monitoring period. The wireless sensor monitoring horizontal displacement data and the manual monitoring horizontal displacement data reflect the parabolic deformation trend of the foundation pit supporting wall. The horizontal displacement monitoring of the foundation pit support by the wireless vibration inclination sensor is very close to the displacement monitoring of the point position at the bottom of the wall by the manual inclination monitoring. The horizontal displacement monitoring data of the wireless vibration inclination sensor at the middle and upper points is larger than that of the manual monitoring data. The maximum difference appeared at the point of 3 meters on March 27, and the difference reached 2.5. During the monitoring period, the average difference between the horizontal displacement monitoring and the artificial inclination monitoring data of the wireless vibration inclination sensor on the upper plane of the plane is monitored.

The horizontal displacement monitoring of the foundation of the wireless vibration inclination sensor is different from that of the manual inclination monitoring. Wireless monitoring directly monitors the deformation of the support structure. Manual monitoring indirectly reflects the deformation of the support structure by monitoring the displacement of the soil outside the support structure.

Therefore, wireless monitoring can directly reflect the deformation of the support structure.

\subsection{Application of wireless monitoring technology in tunnel engineering}

Nanjing Metro Line 4 belongs to the Lower Yangtze Fault Block tectonic unit area of the Yangtze fault block area in the regional earth structure.

The geological structure of the area is complex.

The Nanjing area was activated by the Yanshanian period, and several secondary tectonic units were developed. The folds were seriously damaged by the structure. The NE-trending compression torsion and the NW-trending transverse fracture are relatively developed. The depressions and fault depressions control the developmental distribution of the thick Mesozoic strata.

The section pipe loop of the shield of Dongliu Station to Qinglong Station of Nanjing Metro Line 4 consists of 6 segments, which are K, A1, A2, B1 and B2 blocks respectively. The adjacent two ring segments of the shield tunnel of Nanjing Metro Line 4 are in a staggered arrangement. In the application of underground engineering, two shield ring segments were selected for modeling. The segment finite element model is in the form of a twodimensional grid. The joint part of the segment of the model adopts the equivalent processing method of weakening stiffness. As for the pipe segment joints within the ring, a $0.08 \mathrm{~m}$ circumference pipe segment mesh is cut from the pipe segment joints in this model to be treated as the joint part. Since the segment joint portion is mainly subjected to a bending moment, the modified form is equivalent to the cantilever beam of the segment joint portion.

Analysis of on-site monitoring results: A total of five wireless network nodes and one gateway were installed in the monitoring section of the shield section of the East Line Station to the Qinglong Station of Nanjing Metro Line 4. Among them, four wireless inclinometers are used to measure tunnel deformation. They are wireless vibrating tilt sensor nodes 36, 38, 42 and wireless retrofit inclinometer node ports, respectively. One temperature sensor is used to measure environmental humidity, and one gateway node is connected to the computer. The closer nodes transmit the data directly to the gateway. Distant nodes are uploaded to the gateway via relay nodes.

For the horizontal displacement monitoring of foundation pit supporting structure and the deformation monitoring of shield tunnel structure based on wireless vibration inclination sensor, the foundation pit project of the Teaching and Research Building of the School of Transportation of Southeast University and the shield tunnel project of the Dongliu Station to the Qinglong Station of Nanjing Metro Line 4 were studied. Through numerical simulation and on-site monitoring, the following conclusions are drawn:

The horizontal displacement deformation of the foundation pit wall into the pit direction gradually increases with the excavation degree of the foundation pit. The horizontal deformation of the retaining wall at the test point of the horizontal displacement monitoring test of the foundation pit support structure with the wireless vibration inclination sensor is presented as a parabolic line. 
The measured section has a small horizontal displacement at the bottom and top ends of the foundation pit. The overall deformation trend of foundation pit support structure at the horizontal displacement monitoring section is parabolic. The test foundation pit support structure has a large horizontal displacement in the middle of the horizontal displacement monitoring section. At the monitoring section -3 meters, the horizontal displacement reached 3.46 on April 7. The deep foundation pit support has a cumulative horizontal displacement of $59 \mathrm{~mm}$ during the three-week monitoring period.

The horizontal displacement monitoring of the foundation pit line inclination sensor of the engineering foundation pit and the manual inclination monitoring are very close to the displacement monitoring values at the bottom of the wall. In the upper middle point, the wireless vibration tilt sensor horizontal displacement monitoring data has a larger horizontal displacement than the manual monitoring data. During the monitoring period, the average difference between the horizontal displacement monitoring and the artificial inclination monitoring data of the upperpoint wireless vibration inclination sensor of the monitoring plane is about $1 \mathrm{~mm}$. The monitoring object of horizontal displacement of foundation pit support by wireless vibration inclination sensor is different from that by manual inclination measurement. Wireless monitoring directly monitors the deformation of the support structure. However, manual monitoring indirectly reflects the deformation of the supporting structure by monitoring the displacement of the outer working body of the supporting structure. Wireless monitoring can more directly reflect the deformation of the supporting structure.

The convergence monitoring data of the shield tunnel project from the Dongliu Station to the Qinglong Station of Nanjing Metro Line 4 shows that during the construction process, the transverse pipe diameter of the monitoring pipe section shows an expansion trend. After the lateral expansion reaches the peak, the expansion value of shield tunneling declines. The maximum value of transverse diameter expansion in field test data is $6.0 \mathrm{~mm}$. The deformation trend and deformation amount of the monitoring data are similar to the numerical simulation results.

The field monitoring results of the wireless sensor of the shield tunnel project from the Dongliu Station to the Qinglong Station of Nanjing Metro Line 4 show that the signal transmission of the wireless sensor is stable during the on-site monitoring activities of the shield tunnel project. The monitoring data is continuous, and the data loss rate is very low, which can fully meet the deformation monitoring requirements of the shield tunnel lining.

\section{Numerical Simulation Analysis of Deep Foundation Pit Support Structure}

\subsection{Introduction of Midas/GTS software}

Numerical simulation analysis is the science of solving mathematical problems with the help of computer. According to the actual project, the numerical simulation analysis in geotechnical engineering uses computer programs to establish an analytical model that conforms to the actual engineering. Additional conditions such as weight, load, etc. are applied to the model. Various geotechnical conditions and fields can be analyzed under different load and boundary conditions.

Mathematical methods such as finite element method and difference method are used to calculate the deformation and stress analysis methods of the model. The selection of parameters and the selection of boundary conditions will directly affect the accuracy of the calculation results.

Midas/GT software uses intuitive 3D modeling techniques. It includes a variety of geometric modeling functions, ground generators based on survey data, automatic generation of coupled shared surfaces, multiple material models and meshing methods, construction stage modeling assistant, a variety of analysis type assistants and other fast and efficient modeling processing methods. The default milti-frontal solver of the software can largely improve the analytical performance of the software. With excellent analysis mode and analysis speed, the software is especially suitable for building structural models of wall subdivision. This provides more reliable results for the calculation of complex projects. The convenient model post-processor can use animated visual display and three-dimensional space cut-off function to check the calculation results and confirm the validity of the results. Model information and analysis results are automatically output. Midas/GTS is a practical software for quick and easy modeling and query results. This software is widely used in geotechnical engineering.

The analysis of the construction stage is the analysis of the geotechnical construction process. The simulation analysis of the construction phase of the software is an additive process. Before the analysis of the construction stage, the important construction conditions are simplified and extracted. Initial geostress engineering generally performs initial stress analysis. The initial load condition is displacement reset. Through the initial stress analysis, important parameters such as the nonlinear characteristics of the material and the shear strength of the soil can be obtained. The internal force of the newly added unit is zero, that is to say, the newly added unit can be added at any stage, which is 
conducive to inspection, modification and construction. When the unit in the last construction phase is passivated, the internal force of the unit is allotted to the adjacent unit. The above method can make the simulation of the construction stage simple and rapid. Because the construction phase simulation is an additive process, the design of the previous phase directly affects the subsequent computational simulation. Therefore, the construction phase is reasonably simplified.

The excavation process during the construction phase is simulated as much as possible. This is the key to the analysis of the construction phase.

\subsection{Basic assumptions for the Midas/GTS construction phase simulation}

Due to the complexity of the project and the limitations of the finite element simulation, it is impossible to perform a 1:1 identical finite element simulation of the foundation pit excavation.

According to the specific conditions of the project, the construction conditions are simplified and numerical simulation analysis is carried out.

Assumptions were put forward before the simulation as follows:

The soil in the simulated section is evenly distributed without overlapping parts, and $\mathrm{m}-\mathrm{c}$ elastoplastic constitutive model is adopted for the soil.

According to the actual working conditions, external precipitation is carried out on both sides of the foundation pit, and the underground water level of the site is always kept below $2 \mathrm{~m}$ of the designed level of the bottom of the pit. Groundwater effects are not considered in the simulation calculations.

The stiffness of the support structure material in the model is much greater than that of the soil. Through the monitoring of the horizontal displacement of the retaining pile and the axial force of the steel support, it can be seen that the force and deformation of the supporting structure are far less than the ultimate bearing capacity of the structure.

Therefore, the elastic beam unit is used for the simulation analysis of the steel support and the lattice column.

Due to the large amount of traffic on the construction section, it is impossible to carry out closed road construction. Only the middle 6 lanes of the original 8 lanes can be used for construction. A half-stack bridge is constructed at the joint of the upper crown and beam at the east side of the foundation pit for stacking construction materials. The main function of the lattice column in the paving system is to provide vertical bearing capacity for the trestle plate.

Therefore, only the first support concrete and the longitudinal contact beam are simulated.
The foundation pit adopts a half cover digging method. On the trestle board, construction equipment is temporarily stacked. Dynamic load factors such as cranes cannot be accurately considered.

This simulation uses ground overload $\mathrm{q}=22.5 \mathrm{kN} / \mathrm{m}^{2}$.

\subsection{Division of the mesh of the model}

Meshing is an important part of building a finite element model. Reasonable meshing can directly affect the accuracy of the simulation results. Model meshing is divided by a three-dimensional hybrid mesh generator. The ground-connected wall, crown beam and cofferdam model use the extraction unit command to establish the friction surface unit between different materials.

The parameter control of the soil and support structure is carried out before the division.

The specific control parameters are as follows: the unit of the lattice column is 1 , the unit of the soil boundary is 3 , and the unit of the excavation soil in the foundation pit is 1 . When the soil in the foundation pit is meshed, the influence of the internal lattice column on the adjacent excavation soil is considered.

After the meshing is completed, the mesh topology is checked.

The locking unit does not exist.

This indicates that the higher mesh quality is conducive to the convergence of subsequent calculation results.

\section{Comparative Analysis of Numerical Simulation and Measured Data}

\subsection{Comparative analysis of steel support axial force simulation results and measured data}

The Midas/GTS software performs numerical simulations of deep foundation pits.

The transverse steel support is simulated by a one-dimensional beam unit.

The variation trend of the support axial force of the simulated results is basically the same as that of the measured values.

The axial force of the second steel support increased steadily.

The third steel support is the main lateral support structure of the foundation pit retaining structure, the axial force value is the largest, and the steel support axial force tends to be gentle.

The simulated values of the steel support axial force under different working conditions are compared with the measured values. 


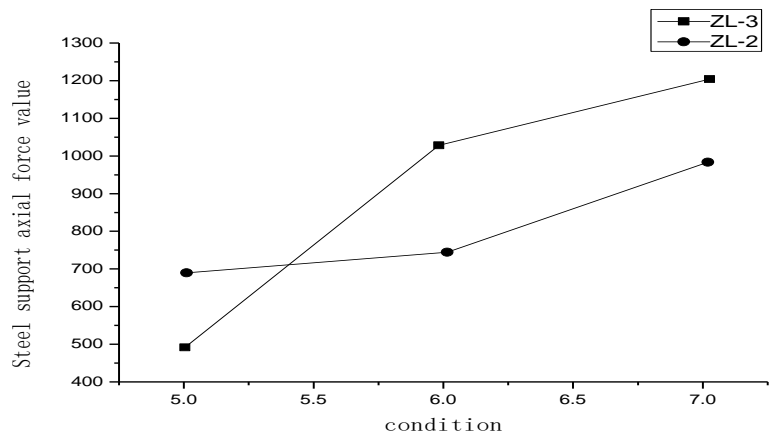

Figure 5: Simulated structure and measured curve of axial force of steel support under different working conditions

Figure 5 shows the change curve of the simulated and measured axial forces of steel supports. The variation trend of the support axial force of the simulated results is basically the same as that of the measured values. The axial force of the second steel support increased steadily. The third steel support is the main lateral support structure of the foundation pit retaining structure, and the axial force value is the largest. The axial force of the steel support tends to be gentle. The simulated and measured values of axial force of steel support under different working conditions are compared. The difference value is the value of the corresponding measured result minus the simulated result.

By comparing and analyzing the difference between the measured and simulated values, the simulation results and the measured results can be found. Except for the working condition 8, the difference between the two is small. It is generally about $10 \%$. The differences under each working condition are analyzed separately. It can be found that the simulation results are generally larger than the measured results. The deeper the excavation, the greater the difference. Because of the complexity of rock and soil mass, the force of support structure becomes more complex with the deeper excavation.

It can be concluded that the calculation model can well simulate the stress change of steel support with the progress of foundation pit excavation. In the simulation analysis of the foundation pits in the trans-season construction in Northeast China, the influence of temperature changes on the supporting structure should be considered. The axial force simulation value of the steel support is used to simulate the structure, and the monitoring results of the foundation pit are predicted and judged.

\subsection{Comparative analysis of deformation simulation results and measured data of envelope structure}

The cast-in-place pile in the retaining structure adopts the principle of equal stiffness conversion, and the calculation is simplified by replacing the retaining pile with the ground wall. The ground wall uses a two-dimensional plate unit in the numerical simulation. The geometric disjunctive surface method is adopted. The horizontal displacement of the ground wall is represented by DisplacementTXTRANSLATION(V).

The numerical simulation results of the deformation of the retaining structure are basically the same as the change trend of the field monitoring value of WB12-14D pile deformation. They are all bow-shaped deformations with large ends at the middle. The horizontal displacement of the pile top is about $4 \mathrm{~mm}$, which indicates that the crown beam and the first concrete support can effectively control the displacement of the top of the retaining pile. The displacement of the bottom of the pile is about $1 \mathrm{~mm}$, which proves that the bottom end of the envelope structure meets the requirements. The maximum deformation of the envelope structure of the simulated structure is $9.50 \mathrm{~mm}$, the measured value is $10.02 \mathrm{~mm}$, and the error rate is $5 \%$. Numerical simulation can well reflect the deformation trend of the retaining structure in the process of foundation pit excavation. The simulation results of the horizontal displacement of $0.5 \mathrm{H}$ retaining pile under different working conditions are compared with the measured values.

The simulation results of the retaining piles at $0.5 \mathrm{H}$ are larger than the measured results. In the numerical simulation, the envelope structure is deformed into a bow shape. The actual monitoring data show that the deformation of the enclosure structure first changes from "forward-inclined" to "bow-shaped" with large middle ends and small middle ends. In numerical simulation, the excavation of foundation pit soil is a one-time complete release of the stress of the excavated element. The actual project is gradually unloaded with the construction, so it has a great impact on the construction conditions in the previous period. When the deformation of the foundation pit is excessive, the difference between the two is rapidly reduced. In summary, the calculation model can simulate the change of the retaining pile during the foundation pit excavation process.

\subsection{Influence of pre-axial force on supporting structure}

Steel support is an ideal elastoplastic material.

The steel support structure has the advantages of easy installation and disassembly, and can be recycled. The biggest advantage is that it can apply pre-stress. The pre-stressing of the steel support is an active support measure that eliminates the slack in the support system and allows the steel support to act quickly and actively. The shear stress accumulated in the soil during excavation is reduced, 
and the deformation of the envelope structure is improved.

The inevitable construction gap between the steel support and the fence is eliminated.

The safety performance of the enclosure is increased. In comparison, the curing time of concrete supporting structure is too long.

During this time, the enclosure structure will be greatly deformed.

This support compression deformation will adversely affect the surrounding structure and affect the overall construction schedule.

Only when the compression is deformed to a certain amount, the cross-sectional resistance advantage of the concrete support is shown.

Combined with the material properties of the concrete and the deformation law of the strip foundation retaining structure, the concrete support should be designed as the first inner support of the deep foundation pit project, that is, the top of the envelope, or the location of the larger deformation of the envelope.

At present, in the strip type deep foundation pit engineering, the value of the pre-axial force of the steel support is customarily taken as about $50 \%$ of the design value of the steel support axial force.

The conventional choice does not take into account the complexity of the construction environment.

At present, it is difficult to calculate the exact value of steel support force under different construction stages through theoretical calculation, and the single standard stipulated on this basis lacks scientific basis.

The design value of pre-axial force of steel support in this project is $400 \mathrm{kN}, 400 \mathrm{kN}, 500 \mathrm{kN}$ from top to bottom, respectively, accounting for $11.3 \%$, $14.2 \%$ and $14.2 \%$ of the ultimate bearing capacity of steel. It can be seen that the value of the pre-axial force is very conservative.

On this basis, the pre-axial force value of the steel support is increased to reduce the deformation of the envelope structure.

This is of great significance to the construction of the project.

On the basis of the original analysis model, by changing the pre-axial force value of the steel support, the deformation of the ground wall and the force change of the support structure are compared.

The optimal steel support pre-axial force value is obtained, which provides a basis for the reasonable value of the pre-axial force.

Comparison of final axial forces of different prestressed steel supports is shown in Figure 6.

Comparison of final deformation of different prestressed retaining piles is shown in Figure 7.

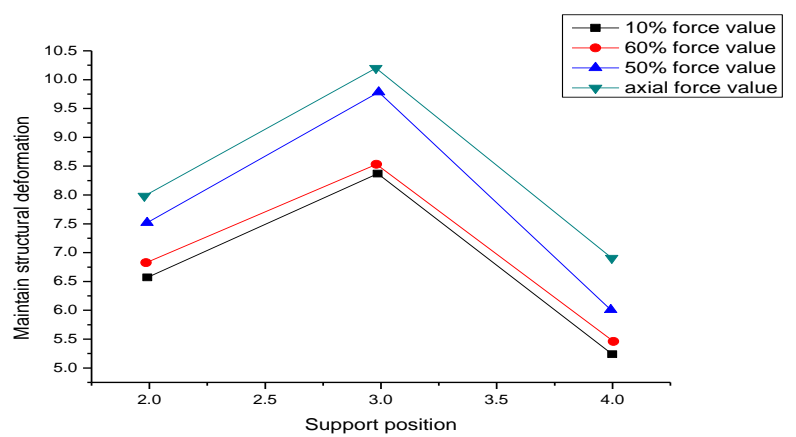

Figure 6: Comparison of final axial forces of different prestressed steel supports

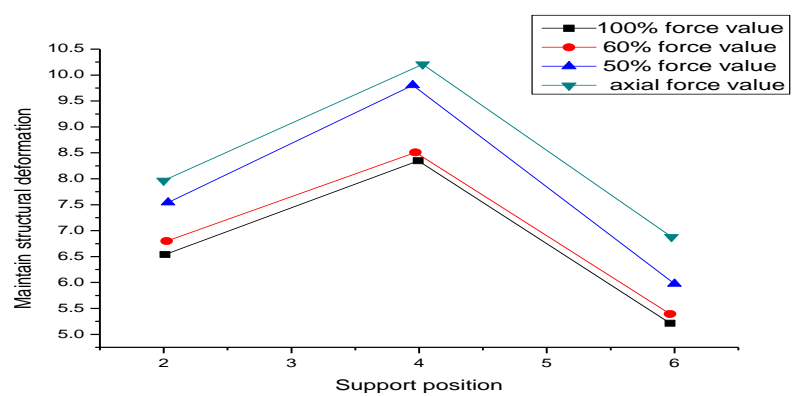

Figure 7: Comparison of final deformation of different prestressed retaining piles

It can be seen that when the prestress value of the steel support is increased, the axial force value of the steel support increases, and the deformation of the envelope structure decreases. The pre-axial force of the steel support has little effect on the final axial force value of the steel support. The trend of the curves in Figure 6 - Figure 7 is basically the same, but the numerical difference is obvious.

The variation law of the influence of the $50 \%$, $60 \%$ and $70 \%$ steel support axial force design values on the deformation of the retaining pile was observed. It can be clearly seen that the $60 \%$ design value of the envelope structure deformation is most obvious. However, the prestress value continues to increase, and the axial force of the steel support increases significantly, but the deformation of the retaining pile does not continue to decrease. It is concluded that $60 \%$ of the preloaded axial force can better control the deformation of the enclosure structure, thus increasing the safety and stability of the structure.

The Midas/GTS finite element software is used to numerically simulate the tunnel in the southern section of the Shenyang-Northern Expressway. The stress and displacement of the foundation pit soil were analyzed as a whole. By comparing and analyzing the numerical simulation results of the axial force of steel support and the deformation of the surrounding structure with the measured data, the results are as follows: 
The variation of the support axial force value and the measured value in the simulation results are basically the same, and the values are basically consistent. The correctness and validity of the monitoring data and numerical simulation results of this project were verified. In the actual engineering design, the above software was used for simulation. The optimal support structure system is obtained to maximize cost savings. In addition, finite element simulation can effectively predict the deformation and stress of the supporting structure, and give some guidance for design and construction. Combined with the measured data and numerical simulation results, the steel support can effectively control the deformation of the envelope structure.

When the steel support is erected and $60 \%$ of the design axial force value is added, the deformation of the retaining structure can be better reduced. Furthermore, the safety and stability of the structure is increased.

\section{Conclusion}

Based on the tunnel in the southern section of the expressway project, the methods of monitoring, theoretical analysis and numerical simulation of the foundation pit support structure are combined. The stability and safety of the supporting structure system during the construction of deep foundation pits are studied. The main conclusions drawn are as follows:

Based on geological conditions and climate change, a monitoring plan for foundation pit support structure was developed. Automatic monitoring and manual monitoring are combined to efficiently and accurately monitor the deformation of the foundation pit support structure along with the construction progress. This provides a reference for the safe and effective construction of the tunnel in the southern section of the South-North Expressway Project in Shenyang. By comparing the on-site monitoring data of 46 retaining piles, the general deformation of the pile body is $8 \sim 10 \mathrm{~mm}$, which occurs at $1 / 2 \mathrm{H} \sim 2 / 5 \mathrm{H}$ from the ground.

The maximum deformation is $11.65 \mathrm{~mm}$, which accounts for $56 \%$ of the warning value. It shows that the deformation of the retaining structure is effectively controlled throughout the construction process.

With the excavation of the foundation pit, the deformation law of the strip type deep foundation pit retaining pile can be divided into two stages: forward tilt shape and bow shape. The deformation of the retaining pile has obvious space-time effects. With the layered excavation of the foundation pit, the deformation of the retaining structure has an obvious extent. The segmental excavation of spacetime effect can effectively reduce the lateral displacement of the envelope structure.
The axial force of three steel supports in the same section increases with the increase of excavation depth. It grows in steps. Foundation pit excavation has the greatest influence on the axial force of the steel support. The width of the foundation pit and the way of excavation are the main factors affecting the internal force and stability of the steel support.

By comparing and analyzing the numerical simulation results and the on-site monitoring values, it is concluded that the deformation of the retaining structure and the stress value of the steel support are basically consistent with the progress of the construction.

The correctness and validity of engineering monitoring data and numerical simulation results were verified. The Midas/GTS software can be used to analyze the supporting structure system of similar projects.

The deformation trend of the retaining structure and the axial force of the steel support in the same section are obtained to guide the construction.

The measured data and numerical simulation results were comprehensively analyzed. For the combined support system of steel support and retaining pile, there is a coordination between deformation and force.

The steel support can effectively control the deformation of the envelope structure.

When the steel support is erected and $60 \%$ of the design axial force value is added, the deformation of the retaining structure can be better reduced.

Furthermore, the safety and stability of the structure is increased.

\section{Acknowledgement}

Research startup subject of Yangtze Normal Universi ty: 2017KYQD16

\section{References}

[1] Li, H., \& Ou, J. (2016). The state of the art in structural health monitoring of cable-stayed bridges. Journal of Civil Structural Health Monitoring, 6(1), 43-67.

[2] Zhang, B., Zhang, L., Deng, W., Jin, L., Chun, F., Pan, H., ... \& Wang, Z. L. (2017). Self-powered acceleration sensor based on liquid metal triboelectric nanogenerator for vibration monitoring. ACS nano, 11(7), 7440-7446.

[3] Huang, Z., Fu, H., Chen, W., Zhang, J., \& Huang, H. (2018). Damage detection and quantitative analysis of shield tunnel structure. Automation in Construction, 94, 303-316.

[4] Zhou, Y., Su, W., Ding, L., Luo, H., \& Love, P. E. (2017). Predicting safety risks in deep foundation pits in subway infrastructure projects: support vector machine approach. 
Journal of Computing in Civil Engineering, 31(5), 04017052.

[5] Hu, Q. J., Xu, Y. H., Long, Z. W., \& Zaxi, P. X. (2015). Impact analysis of deep foundation pit excavation on adjacent pipeline in the sand gravel area. Electronic Journal of Geotechnical Engineering, 20(2), 681-694.

[6] Zhang, G., \& Yan, G. (2015). In-flight simulation of the excavation of foundation pit in centrifuge model tests. Geotechnical Testing Journal, 39(1), 59-68.

[7] Tan, Y., Zhu, H., Peng, F., Karlsrud, K., \& Wei, B. (2017). Characterization of semi-top-down excavation for subway station in Shanghai soft ground. Tunnelling and Underground Space Technology, 68, 244-261.

[8] Xu, Q., Zhu, H., Ma, X., Ma, Z., Li, X., Tang, Z., \& Zhuo, K. (2015). A case history of shield tunnel crossing through group pile foundation of a road bridge with pile underpinning technologies in Shanghai. Tunnelling and Underground Space Technology, 45, 20-33.

[9] Luo, H., \& Gong, P. (2015). A BIM-based code compliance checking process of deep foundation construction plans. Journal of Intelligent \& Robotic Systems, 79(3-4), 549576.

[10] Nikonorov, A., Pavlov, S., Terleev, V., Arefiev, N., Badenko, V., \& Volkova, Y. (2015). Use of enclosing and temporary special structures under the reconstruction of hydraulic facilities in Saint-Petersburg. Procedia Engineering, 117, 258-263.

[11] Zhang, B., Chen, J., Jin, L., Deng, W., Zhang, L., Zhang, H., ... \& Wang, Z. L. (2016). Rotating-diskbased hybridized electromagnetic-triboelectric nanogenerator for sustainably powering wireless traffic volume sensors. ACS nano, $10(6), 6241-6247$

[12] Liang, T. C., Ho, J. F., \& Wu, P. T. (2018). A wireless tilt sensor based on plastic optical fiber and Arduino board microcontroller. Microsystem Technologies, 24(10), 4101-4104.

[13] Hodge, V. J., O'Keefe, S., Weeks, M., \& Moulds, A. (2015). Wireless sensor networks for condition monitoring in the railway industry: A survey. IEEE Trans. Intelligent transportation systems, 16(3), 1088-1106.

[14] Liu, C., Park, J. W., Spencer Jr, B. F., Moon, D. S., \& Fan, J. (2017). Sensor fusion for structural tilt estimation using an acceleration-based tilt sensor and a gyroscope. Smart Materials and Structures, 26(10), 105005.

[15] Bajwa, R., Coleri, E., Rajagopal, R., Varaiya, P., \& Flores, C. (2017). Development of a Cost-Effective Wireless Vibration Weigh-In-Motion System to Estimate Axle Weights of Trucks. Computer-Aided Civil and Infrastructure Engineering, 32(6), 443-457. 